\title{
Use of the paired samples (cerebrospinal fluid and serum) in immunodiagnostic of active and inactive human neurocysticercosis
}

\author{
Ivanildes Solange da Costa Barcelos, Marcelo Simão Ferreira* $/^{+}$, \\ Leandro Pajuaba de Moura*, Germano Francisco Biondi**, Julia Maria Costa-Cruz
}

\author{
Laboratório de Parasitologia, Instituto de Ciências Biomédicas *Departamento de Clínica Médica, Faculdade de Medicina, \\ Universidade Federal de Uberlândia, Av. Pará 1720, 38400-902 Uberlândia, MG, Brasil **Departamento de Veterinária, \\ Higiene e Saúde Pública, Universidade do Estado de São Paulo, Botucatu, SP, Brasil
}

Paired samples of cerebrospinal fluid (CSF) and serum of 30 patients - 10 with active, 10 with inactive neurocysticercosis (NCC), and 10 control subjects - were evaluated by enzyme-linked immunosorbent assay (ELISA) using two Taenia crassiceps metacestode extracts as antigen in order to detect IgG antibodies. In active NCC, high levels of IgG were detected ( $p<0.05$ ). The CSF samples showed $80 \%$ (CI 72-88) of reactivity in the saline extract $(S)$ and 90\% (CI 84-95) in sodium dodecyl sulphate (SDS) and the serum samples were reactive in 90\% (CI 84-95) and 100\% (CI 98-100) in the S and SDS antigenic extracts, respectively. The use of the paired samples of CSF and serum in active NCC showed equivalent results suggesting that the serum samples could be used as a screening in those patients whose CSF puncture is counter-indicated.

Key words: Taenia crassiceps - neurocysticercosis - enzyme-linked immunosorbent assay - serum - cerebrospinal fluid

Neurocysticercosis (NCC) is the most common parasitosis of the central nervous system (CNS) in humans and the infection is acquired through ingestion of eggs from Taenia solium. Signs and symptoms are polymorphic and non-specific, including seizures, focal neurological signs, intra-cerebral hypertension, and cognitive behavioral dysfunctions. Given the wide range of clinical presentation, diagnosis is seldom made by history and physical examination alone. Accurate diagnosis is based on the combination of clinical, epidemiologic, radiographic, and immunologic data (Del Brutto et al. 2001, Hawk et al. 2005).

NCC has been classified in active and inactive forms, according to the associated immune response and the neuroimaging studies (Sotelo et al. 1985). In the active form there are vesicular or degenerating parasites cysts circundated by a strong inflammatory reaction in the cerebral adjacent tissue, but in the inactive form the patient has only calcified lesions and the immune response is minimal (Sotelo et al. 1985, Salgado et al. 1997, Castilho 2004). The cells from inflammatory NCC patients show a predominance of a Th1 immune response upon in vitro stimulation, and a mixed Th1/Th2 cellular immune response in the non-inflammatory phase (Bueno et al. 2004).

Financial support: $\mathrm{CNPq}$

+Corresponding author. E-mail: mferreira@nanet.com.br

Received 31 March 2005

Accepted 2 June 2005
Enzyme-linked immunosorbent assay (ELISA) has been widely employed as a useful tool for the diagnosis of NCC through the detection of T. solium metacestodes IgG antibodies in cerebrospinal fluid (CSF) and serum samples (Costa et al. 1982, White Jr 2000, Hawk et al 2005). The antigens of murine Taenia crassiceps metacestodes, have been used as effective substitutes to the T. solium metacestodes antigens in the detection of IgG in CSF and serum samples due to intense antigenic similarity between both parasites (Vaz et al. 1997, Barcelos et al. 2001, Bragazza et al. 2002, Ishida et al. 2003). A recombinant 10-kDa protein of $T$. solium metacestode (CyDA) was identified as being specific to active NCC and having high homology with T. crassiceps metacestode (Chung et al. 2002).

The aim of this current study was to evaluate the use of paired samples (CSF and serum) in immunodiagnostic of active and inactive forms of human NCC using two $T$. crassiceps metacestodes antigens. This study was approved by the Research Ethics Committee of the Federal University of Uberlândia, Brazil.

A total of 30 paired CSF and serum samples were analyzed and divided into three groups based on clinical and laboratorial data for NCC: (1) active NCC group consisted of 10 samples from patients ( 6 male and 4 female, mean age: 35 years) with signs and symptoms suggestive of NCC and computed tomography and/or magnetic resonance imaging results showing vesicular or degenerative lesions, with or without calcification, in the central nervous system (CNS); (2) inactive NCC group consisted of 10 samples from patients ( 4 male and 6 female, mean age: 31 years) with signs and symptoms compatible with NCC and imaging studies demonstrating only calcifications in the central nervous system; (3) control group consisted 
of 10 samples from patients ( 4 male and 6 female, mean age: 32 years) with other neurological disorders and normal imaging results.

ELISA for the detection of $\mathrm{IgG}$ antibodies was carried out according to Barcelos et al. (2001), using two different antigenic extracts: saline (S) and sodium dodecyl sulphate (SDS), obtained from T. crassiceps metacestodes, and used at a protein concentration of $10 \mu \mathrm{g} / \mathrm{ml}$. CSF samples were tested undiluted and serum samples were diluted 1:200 in phosphate buffered saline (PBS, $0.1 \mathrm{M}, \mathrm{pH}$ 7.2) containing $0.05 \%$ Tween 20 . The conjugate goat IgG anti-human IgGperoxidase (Fc chain specific; Sigma) was diluted at 1:2000. The cut off was established by the mean optical density (OD) obtained from three negative control samples plus two standard deviations. Statistical analysis was performed using the software Statistic for Windows (Stat soft, Inc. 1993) for the comparative analysis between two proportions, considering significance level at $\mathrm{p}<0.05$. A $95 \%$ confidence interval (CI) was stipulated.

The results of ELISA from the three patient groups are showed in the Table, the values of OD were significantly higher in the active NCC than the other two groups ( $\mathrm{p}<$ 0.05 ). The Figure shows the relationship between the values of OD in the CSF and serum samples obtained from patients with active NCC using two antigenic extracts. The results indicate that there were high correlate index using of $\mathrm{S}$ extract.

In the present study, we used the $T$. crassiceps heterologous cysticerci as an alternative source of antigens for the immunological diagnosis of the human NCC. In a previous ELISA study using T. crassiceps $\mathrm{S}$ and SDS antigens in the CSF samples from patients with NCC, Barcelos et al. (2001) demonstrated 85 and $87.5 \%$ of sensitivity and 100 and $97.9 \%$ of specificity, respectively. Pardini et al. (2002), in an ELISA study for detection of IgG antibodies in CSF samples in NCC, showed $100 \%$ of sensitivity and specificity, using antigen extracts obtained from the vesicular fluid of $T$. crassiceps cysticerci and from fractions purified by affinity chromatography with lectin concanavalin A and the glycoprotein antigen separated by electrophoresis.

Serology for NCC can produce false-positive results in samples of patients coming from endemic countries for cysticercosis, such as Brazil, because there is a production of specific antibodies due to previous infections that did not progress for the establishment of metacestodes or because these latter are located outside the neural tis-
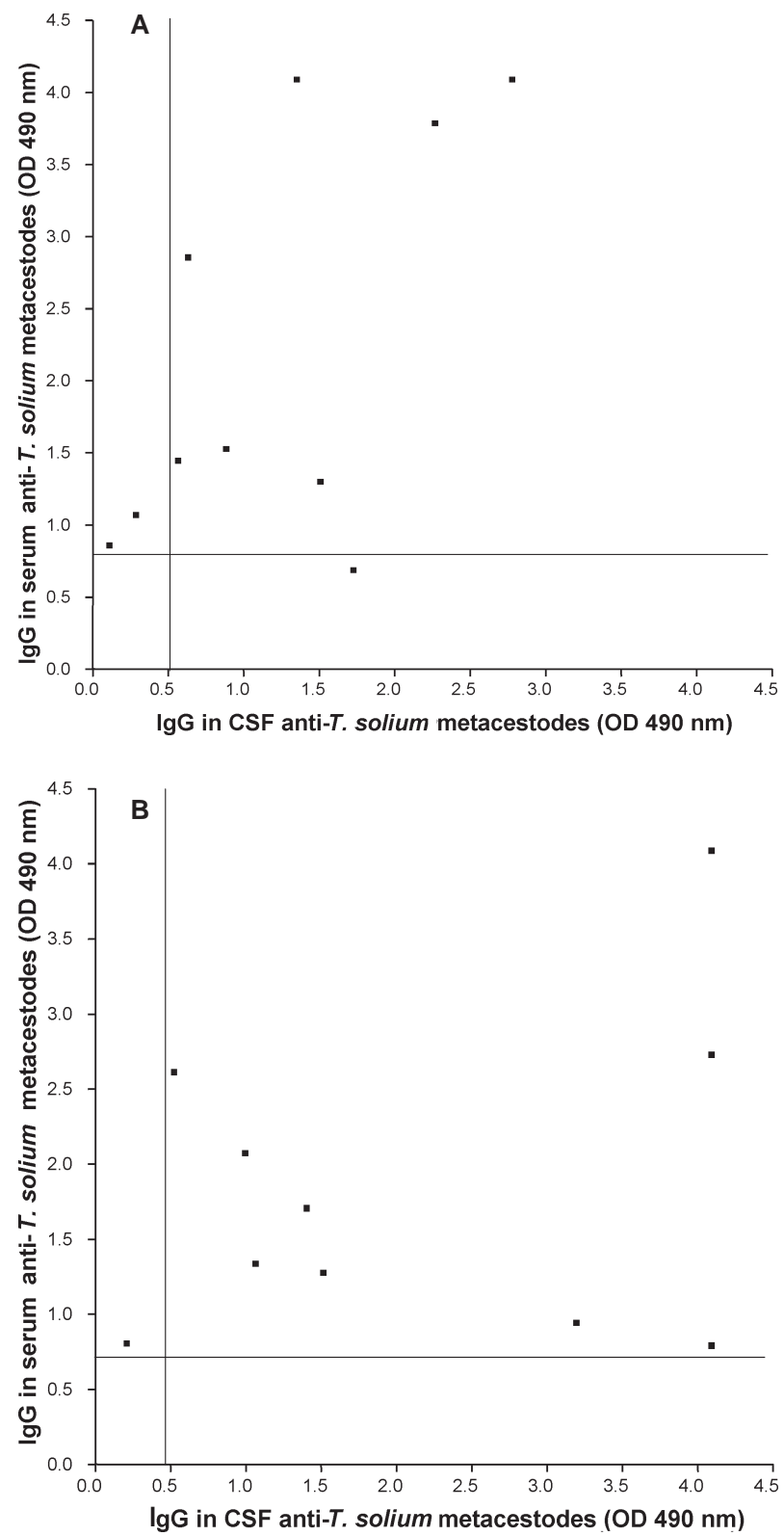

Relationship between the optical density (OD) values of IgG antineurocysticercosis (NCC) in the no diluted cerebrospinal fluid (CSF) and diluted serum samples at 1:200 of 10 patients with the active form of NCC, using Taenia crassiceps metacestodes extracts by ELISA. A: saline (S); B: sodium dodecyl sulphate (SDS) - : cut off values. Pearson values $\mathrm{r}=0.6150(\mathrm{~S})$ and $\mathrm{r}=0.3077$ (SDS).

TABLE

Paired cerebrospinal fluid (CSF) and serum samples in ELISA for the detection of IgG antibodies in human eurocysticercosis (NCC) using two antigenic extracts (saline and sodium dodecyl sulphate) from Taenia crassiceps metacestodes

\begin{tabular}{|c|c|c|c|c|c|c|c|c|c|}
\hline \multirow[b]{3}{*}{ Patient } & \multirow[b]{3}{*}{$n$} & \multicolumn{4}{|c|}{ Saline } & \multicolumn{4}{|c|}{ Sodium dodecyl sulphate } \\
\hline & & \multicolumn{2}{|c|}{ CSF } & \multicolumn{2}{|c|}{ Serum } & \multicolumn{2}{|c|}{ CSF } & \multicolumn{2}{|c|}{ S erum } \\
\hline & & $\%$ & (CI) & $\%$ & (CI) & $\%$ & (CI) & $\%$ & $(\mathrm{CI})$ \\
\hline Active NCC & 10 & 80 & $(72.0-88.0)$ & 90 & $(84.0-95.0)$ & 90 & $(84.0-95.0)$ & 100 & $(98.0-100)$ \\
\hline Inactive NCC & 10 & 0 & & 10 & $(4.0-16.0)$ & 0 & & 20 & $(12.0-28.0)$ \\
\hline Control & 10 & 0 & & 0 & & 0 & & 0 & \\
\hline
\end{tabular}

CI: confidence interval 
sue (Sciutto et al. 2000). The ELISA cross-reactivity among helminthiases was found with the use of antigens (Echinococcus granulosus hydatid fluid, T. solium cysticerci saline extract, and vesicular fluid of $T$. crassiceps) belonging to phylogenetically related parasite species, by sharing same antigenic components (Ishida et al. 2003). By analyzing CSF samples of patients with NCC using excretion/secretion antigens of $T$. solium metacestodes, a significant difference between ELISA results in the detection of $\operatorname{IgG}$ antibodies was shown in order to distinguish the active NCC from the inactive one (Molinari et al. 2002).

The results of this study showed that patients presenting the active form of NCC showed the highest levels of specific IgG antibodies in both samples analyzed, since the immune response is maximized when the parasite goes into the degenerative phases. In the control group analyzed here none of the individuals showed reactivity.

In conclusion the used of the paired samples of CSF and serum in active NCC showed equivalent results suggesting that the serum samples could be used as a screening of those patients whose CSF puncture is contra-indicated.

\section{REFERENCES}

Barcelos ISC, Mineo JR, Silva DAO, Ferreira MS, Moura LP, Biondi GF, Costa-Cruz JM 2001. Detection of IgG in cerebrospinal fluid for diagnosis of neurocysticercosis: evaluation of saline and SDS extracts from Taenia solium and Taenia crassiceps metacestodes by ELISA and immunoblot assay. Trop Med Int Health 6: 219-226.

Bragazza LM, Vaz AJ, Passos ADC, Takayanagui OM, Nakamura PM, Espíndola NM, Pardini A, Bueno EC 2002. Frequency of serum anti-cysticercus antibodies in the population of a rural Brazilian community (Cássia dos Coqueiros, SP) determined by ELISA and immunoblotting using Taenia crassiceps antigens. Rev Inst Med Trop São Paulo 44: 712.

Bueno EC, Machado LR, Livramento JA, Vaz AJ 2004. Cellular immune response of patients with neurocysticercosis (inflammatory and non-inflammatory phases). Acta Trop 91: 205-213.

Castilho M 2004. Immaging of neurocysticercosis. Seminars Roentgenology 39: 465-473.

Chung JY, Yun DH, Eom KS, Kang SY, Kong Y, Cho SY 2002. Taenia solium identification of specific antibody binding regions of metacestode 10-kDa protein. Exp Parasitol 100: 87-94.
Costa JM, Ferreira AW, Makino MM, Camargo ME 1982. Spinal fluid immunoenzymatic assay (ELISA) for neurocysticercosis. Rev Inst Med Trop São Paulo 24: 337-341.

Del Brutto OH, Rajshekhar V, White Jr AC, Tsang VCW, Nash TE, Takayanagui OM, Schantz PM, Evans CAW, Flisser A, Correa D, Botero D, Allan JC, Sarti E, Gonzales AE, Gilman RH, García HH 2001. Proposed diagnostic criteria for neurocysticercosis. Neurology 57: 177-183.

Hawk MW, Shahlaie K, Kim KD, Theis JH 2005. Neurocysticercosis: a review. Surgical Neurol 63: 123-132.

Ishida MMI, Rubinsky-Elefant G, Ferreira AW, HoshinoShimizu S, Vaz AJ 2003. Helminth antigens (Taenia solium, Taenia crassiceps, Toxocara canis, Schistosoma mansoni and Echinococcus granulosus) and cross-reactivities in human infections and immunized animals. Acta Trop 89: 73-84.

Molinari JL, Garcia-Mendoza E, de la Garza Y, Ramirez JA, Sotelo J, Tato P 2002. Discrimination between active and inactive neurocysticercosis by metacestode excretory/secretory antigens of Taenia solium in an enzyme-linked immunosorbent assay. Am J Trop Med Hyg 66: 777-781.

Pardini AX, Peralta RH, Vaz AJ, Machado LR, Peralta JM 2002. Use of Taenia crassiceps cysticercus antigen preparations for detection of antibodies in cerebrospinal fluid samples from patients with neurocysticercosis (Taenia solium). Clin Diag Lab Immunol 9: 190-193.

Salgado P, Rojas R, Sotelo J 1997. Cysticercosis. Clinical classification based on imaging studies. Arch Intern Med 157: 1991-1997.

Sciutto E, Fragoso G, Fleury A, Laclette, JP, Sotelo J, Aluja A, Vargas L, Larralde C 2000. Taenia solium disease in humans and pigs: an ancient parasitosis disease rooted in developing countries and emerging as a major health problem of global dimensions. Microbes Infect 2: 1875-1890.

Sotelo J, Guerrero V, Rubio F 1985. Neurocysticercosis: a new classification based on active and inactive forms. A study of 753 cases. Arch Intern Med 145: 442-445.

Vaz AJ, Nunes CM, Piazza RMF, Livramento JA, Silva MV, Nakamura PM, Ferreira AW 1997. Immunoblot with cerebrospinal fluid from patients with neurocysticercosis using antigen from cysticerci of Taenia solium and Taenia crassiceps. Am J Trop Med Hyg 57: 354-357.

White Jr AC 2000. Neurocysticercosis: updates on epidemiology, pathogenesis, diagnosis and management. Ann Rev Med 51: 197-206. 
